\title{
Automated Security Proofs with Sequences of Games
}

\author{
Bruno Blanchet and David Pointcheval \\ CNRS, École Normale Supérieure, Paris \\ \{blanchet, pointche\}@di.ens.fr
}

\begin{abstract}
This paper presents the first automatic technique for proving not only protocols but also primitives in the exact security computational model. Automatic proofs of cryptographic protocols were up to now reserved to the Dolev-Yao model, which however makes quite strong assumptions on the primitives. On the other hand, with the proofs by reductions, in the complexity theoretic framework, more subtle security assumptions can be considered, but security analyses are manual. A process calculus is thus defined in order to take into account the probabilistic semantics of the computational model. It is already rich enough to describe all the usual security notions of both symmetric and asymmetric cryptography, as well as the basic computational assumptions. As an example, we illustrate the use of the new tool with the proof of a quite famous asymmetric primitive: unforgeability under chosen-message attacks (UF-CMA) of the Full-Domain Hash signature scheme under the (trapdoor)-one-wayness of some permutations.
\end{abstract}

\section{Introduction}

There exist two main frameworks for analyzing the security of cryptographic protocols. The most famous one, among the cryptographic community, is the "provable security" in the reductionist sense [8]: adversaries are probabilistic polynomial-time Turing machines which try to win a game, specific to the cryptographic primitive/protocol and to the security notion to be satisfied. The "computational" security is achieved by contradiction: if an adversary can win such an attack game with non-negligible probability, then a well-defined computational assumption is invalid (e.g., one-wayness, intractability of integer factoring, etc.) As a consequence, the actual security relies on the sole validity of the computational assumption. On the other hand, people from formal methods defined formal and abstract models, the so-called Dolev-Yao 21 framework, in order to be able to prove the security of cryptographic protocols too. However, these "formal" security proofs use the cryptographic primitives as ideal blackboxes. The main advantage of such a formalism is the automatic verifiability, or even provability, of the security, but under strong (and unfortunately unrealistic) assumptions. Our goal is to take the best of each framework, without the drawbacks, that is, to achieve automatic provability under classical (and realistic) computational assumptions. 
The Computational Model. Since the seminal paper by Diffie and Hellman [20], complexity theory is tightly related to cryptography. Cryptographers indeed tried to use $\mathcal{N} \mathcal{P}$-hard problems to build secure cryptosystems. Therefore, adversaries have been modeled by probabilistic polynomial-time Turing machines, and security notions have been defined by security games in which the adversary can interact with several oracles (which possibly embed some private information) and has to achieve a clear goal to win: for signature schemes, the adversary tries to forge a new valid message-signature pair, while it is able to ask for the signature of any message of its choice. Such an attack is called an existential forgery under chosen-message attacks [23. Similarly, for encryption, the adversary chooses two messages, and one of them is encrypted. Then the goal of the adversary is to guess which one has been encrypted [22, with a probability significantly better than one half. Again, several oracles may be available to the adversary, according to the kind of attack (chosen-plaintext and/or chosenciphertext attacks [34 35]). One can see in these security notions that computation time and probabilities are of major importance: an unlimited adversary can always break them, with probability one; or in a shorter period of time, an adversary can guess the secret values, by chance, and thus win the attack game with possibly negligible but non-zero probability. Security proofs in this framework consist in showing that if such an adversary can win with significant probability, within reasonable time, then a well-defined problem can be broken with significant probability and within reasonable time too. Such an intractable problem and the reduction will quantify the security of the cryptographic protocol.

Indeed, in both symmetric and asymmetric scenarios, most security notions cannot be unconditionally guaranteed (i.e. whatever the computational power of the adversary). Therefore, security generally relies on a computational assumption: for instance, the existence of one-way functions, or permutations, possibly trapdoor. A one-way function is a function $f$ which anyone can easily compute, but given $y=f(x)$ it is computationally intractable to recover $x$ (or any pre-image of $y$ ). A one-way permutation is a bijective one-way function. For encryption, one would like the inversion to be possible for the recipient only: a trapdoor one-way permutation is a one-way permutation for which a secret information (the trapdoor) helps to invert the function on any point.

Given such objects, and thus computational assumptions about the intractability of the inversion (without trapdoors), we would like that security could be achieved without any additional assumptions. The only way to "formally" prove such a fact is by showing that an attacker against the cryptographic protocol can be used as a sub-part in an algorithm (the reduction) that can break the basic computational assumption.

Observational Equivalence and Sequence of Games. Initially, reductionist proofs consisted in presenting a reduction, and then proving that the view of the adversary provided by the reduction was (almost) indistinguishable to the view of the adversary during a real attack. Such an indistinguishability was quite technical and error-prone. Victor Shoup [37] suggested to prove it by small changes [1], using a "sequence of games" (a.k.a. the game hopping technique) that the 
adversary plays, starting from the real attack game. Two consecutive games look either identical, or very close to each other in the view of the adversary, and thus involve a statistical distance, or a computational one. In the final game, the adversary has clearly no chance to win at all. Actually, the modifications of games can be seen as "rewriting rules" of the probability distributions of the variables involved in the games. They may consist of a simple renaming of some variables, and thus to perfectly identical distributions. They may introduce unlikely differences, and then the distributions are "statistically" indistinguishable. Finally, the rewriting rule may be true under a computational assumption only: then appears the computational indistinguishability.

In formal methods, games are replaced with processes using perfect primitives modeled by function symbols in an algebra of terms. "Observational equivalence" is a notion similar to indistinguishability: it expresses that two processes are perfectly indistinguishable by any adversary. The proof technique typically used for observational equivalence is however quite different from the one used for computational proofs. Indeed, in formal models, one has to exploit the absence of algebraic relations between function symbols in order to prove equivalence; in contrast to the computational setting, one does not have observational equivalence hypotheses (i.e. indistinguishability hypotheses), which specify security properties of primitives, and which can be combined in order to obtain a proof of the protocol.

Related Work. Following the seminal paper by Abadi and Rogaway [1, recent results 32 18 25] show the soundness of the Dolev-Yao model with respect to the computational model, which makes it possible to use Dolev-Yao provers in order to prove protocols in the computational model. However, these results have limitations, in particular in terms of allowed cryptographic primitives (they must satisfy strong security properties so that they correspond to Dolev-Yao style primitives), and they require some restrictions on protocols (such as the absence of key cycles).

Several frameworks exist for formalizing proofs of protocols in the computational model. Backes, Pfitzmann, and Waidner 56.3 have designed an abstract cryptographic library and shown its soundness with respect to computational primitives, under arbitrary active attacks. Backes and Pfitzmann [4] relate the computational and formal notions of secrecy in the framework of this library. Recently, this framework has been used for a computationally-sound machinechecked proof of the Needham-Schroeder-Lowe protocol [38]. Canetti [16] introduced the notion of universal composability. With Herzog [17, they show how a Dolev-Yao-style symbolic analysis can be used to prove security properties of protocols within the framework of universal composability, for a restricted class of protocols using public-key encryption as only cryptographic primitive. Then, they use the automatic Dolev-Yao verification tool ProVerif 12 for verifying protocols in this framework. Lincoln, Mateus, Mitchell, Mitchell, Ramanathan, Scedrov, and Teague [29/30/31/36/33] developed a probabilistic polynomial-time calculus for the analysis of cryptographic protocols. Datta et al [19] have designed a computationally sound logic that enables them to prove computational 
security properties using a logical deduction system. These frameworks can be used to prove security properties of protocols in the computational sense, but except for [17. which relies on a Dolev-Yao prover, they have not been automated up to now, as far as we know.

Laud [26] designed an automatic analysis for proving secrecy for protocols using shared-key encryption, with passive adversaries. He extended it [27] to active adversaries, but with only one session of the protocol. This work is the closest to ours. We extend it considerably by handling more primitives, a variable number of sessions, and evaluating the probability of an attack. More recently, he 28 designed a type system for proving security protocols in the computational model. This type system handles shared- and public-key encryption, with an unbounded number of sessions. This system relies on the Backes-PfitzmannWaidner library. A type inference algorithm is sketched in [2].

Barthe, Cerderquist, and Tarento [7/39] have formalized the generic model and the random oracle model in the interactive theorem prover Coq, and proved signature schemes in this framework. In contrast to our specialized prover, proofs in generic interactive theorem provers require a lot of human effort, in order to build a detailed enough proof for the theorem prover to check it.

Halevi 24 explains that implementing an automatic prover based on sequences of games would be useful, and suggests ideas in this direction, but does not actually implement one.

Our prover, which we describe in this paper, was previously presented in 1314, but in a more restricted way. It was indeed applied only to classical, Dolev-Yao-style protocols of the literature, such as the Needham-Schroeder public-key protocol. In this paper, we show that it can also be used for the proof of security of cryptographic primitives. [13:14] considered only asymptotic proofs. In this paper, we have extended the prover for providing exact security proofs. We also extend it to the proof of authentication properties, while 1314 considered only secrecy properties. Finally, we also show how to model a random oracle.

Achievements. As in 1314, our goal is to fill the gap between the two usual techniques (computational and formal methods), but with a direct approach, in order to get the best of each: a computationally sound technique, which an automatic prover can apply. More precisely, we adapt the notion of observational equivalence so that it corresponds to the indistinguishability of games. To this aim, we also adapt the notion of processes: our processes run in time $t$ and work with bit-strings. Furthermore, the process calculus has a probabilistic semantics, so that a measure can be defined on the distinguishability notion, or the observational equivalence, which extends the "perfect indistinguishability": the distance between two views of an adversary. This distance is due to the application of a transformation, which is purely syntactic. The transformations are rewriting rules, which yield a game either equivalent or almost equivalent under a "computational assumption". For example, we define a rewriting rule, which is true under the one-wayness of a specific function. The automatic prover tries to apply the rewriting rules until the winning event, which is executed in the 
original attack game when the adversary breaks the cryptographic protocol, has totally disappeared: the adversary eventually has a success probability 0 . We can then upper-bound the success probability of the adversary in the initial game by the sum of all gaps.

Our prover also provides a manual mode in which the user can specify the main rewriting steps that the prover has to perform. This allows the system to prove protocols in situations in which the automatic proof strategy does not find the proof, and to direct the prover towards a specific proof, for instance a proof that yields a better reduction, since exact security is now dealt with.

\section{A Calculus for Games}

\subsection{Description of the Calculus}

In this section, we review the process calculus defined in [1314] in order to model games as done in computational security proofs. This calculus has been carefully designed to make the automatic proof of cryptographic protocols easier. One should note that the main addition from previous models 3328 is the introduction of arrays, which allow us to formalize the random oracle model [9], but also the authenticity (unforgeability) in several cryptographic primitives, such as signatures, message authentication codes, but also encryption schemes. Arrays allow us to have full access to the whole memory state of the system, and replace lists often used in cryptographic proofs. For example, in the case of a random oracle, one generally stores the input and output of the random oracle in a list. In our calculus, they are stored in arrays.

Contrarily to 1314, we adopt the exact security framework 10], instead of the asymptotic one. The cost of the reductions, and the probability loss will thus be precisely determined. We also adapt the syntax of our calculus, in order to be closer to the usual syntax of cryptographic games.

In this calculus, we denote by $T$ types, which are subsets of bitstring $\perp_{\perp}=$ bitstring $\cup\{\perp\}$, where bitstring is the set of all bit-strings and $\perp$ is a special symbol. A type is said to be fixed-length when it is the set of all bit-strings of a certain length. A type $T$ is said to be large when its cardinal is large enough so that we can consider collisions between elements of $T$ chosen randomly with uniform probability quite unlikely, but still keeping track of the small probability. Such an information is useful for the strategy of the prover. The boolean type is predefined: bool $=\{$ true, false $\}$, where true $=1$ and false $=0$.

The calculus also assumes a finite set of function symbols $f$. Each function symbol $f$ comes with a type declaration $f: T_{1} \times \ldots \times T_{m} \rightarrow T$. Then, the function symbol $f$ corresponds to a function, also denoted $f$, from $T_{1} \times \ldots \times T_{m}$ to $T$, such that $f\left(x_{1}, \ldots, x_{m}\right)$ is computable in time $t_{f}$, which is bounded by a function of the length of the inputs $x_{1}, \ldots, x_{m}$. Some predefined functions use the infix notation: $M=N$ for the equality test (taking two values of the same type $T$ and returning a value of type bool), $M \wedge N$ for the boolean and (taking and returning values of type bool). 
Let us now illustrate on an example how we represent games in our process calculus. As we shall see in the next sections, this example comes from the definition of security of the Full-Domain Hash (FDH) signature scheme 9]. This example uses the function symbols hash, pkgen, skgen, $\mathrm{f}$, and invf (such that $x \mapsto \operatorname{invf}(s k, x)$ is the inverse of the function $x \mapsto \mathrm{f}(p k, x))$, which will all be explained later in detail. We define an oracle Ogen which chooses a random seed $r$, generates a key pair $(p k, s k)$ from this seed, and returns the public key $p k$ :

$$
\operatorname{Ogen}():=r \stackrel{R}{\leftarrow} \text { seed } ; p k \leftarrow \operatorname{pkgen}(r) ; s k \leftarrow \operatorname{skgen}(r) ; \operatorname{return}(p k)
$$

The seed $r$ is chosen randomly with uniform probability in the type seed by the construct $r \stackrel{R}{\leftarrow}$ seed. (The type seed must be a fixed-length type, because probabilistic bounded-time Turing machines can choose random numbers uniformly only in such types. The set of bit-strings seed is associated to a fixed value of the security parameter.)

Next, we define a signature oracle $O S$ which takes as argument a bit-string $m$ and returns its FDH signature, computed as $\operatorname{invf}(s k$, hash $(m))$, where $s k$ is the secret key, so this oracle could be defined by

$$
O S(m: \text { bitstring }):=\operatorname{return}(\operatorname{invf}(s k, \operatorname{hash}(m)))
$$

where $m$ : bitstring means that $m$ is of type bitstring, that is, it is any bitstring. However, this oracle can be called several times, say at most $q S$ times. We express this repetition by foreach $i S \leq q S$ do $O S$, meaning that we make available $q S$ copies of $O S$, each with a different value of the index $i S \in[1, q S]$. Furthermore, in our calculus, variables defined in repeated oracles are arrays with a cell for each call to the oracle, so that we can remember the values used in all calls to the oracles. Here, $m$ is then an array indexed by $i S$. Along similar lines, the copies of the oracle $O S$ itself are indexed by $i S$, so that the caller can specify exactly which copy of $O S$ he wants to call, by calling $O S[i S]$ for a specific value of $i S$. So we obtain the following formalization of this oracle:

foreach $i S \leq q S$ do $O S[i S](m[i S]:$ bitstring $):=\operatorname{return}(\operatorname{invf}(s k, \operatorname{hash}(m[i S])))$

Note that $s k$ has no array index, since it is defined in the oracle Ogen, which is executed only once.

We also define a test oracle $O T$ which takes as arguments a bit-string $m^{\prime}$ and a candidate signature $s$ of type $D$ and executes the event forge when $s$ is a forged signature of $m^{\prime}$, that is, $s$ is a correct signature of $m^{\prime}$ and the signature oracle has not been called on $\mathrm{m}^{\prime}$. The test oracle can be defined as follows:

$$
\begin{aligned}
& O T\left(m^{\prime}: \text { bitstring, } s: D\right):=\text { if } \mathrm{f}(p k, s)=\operatorname{hash}\left(m^{\prime}\right) \text { then } \\
& \text { find } \left.u \leq q S \text { suchthat (defined }(m[u]) \wedge m^{\prime}=m[u]\right) \text { then end } \\
& \text { else event forge }
\end{aligned}
$$

It first tests whether $\mathrm{f}(p k, s)=\operatorname{hash}\left(m^{\prime}\right)$, as the verification algorithm of FDH would do. When the equality holds, it executes the then branch; otherwise, it 
executes the else branch which is here omitted. In this case, it ends the oracle, as if it executed end. When the test $\mathrm{f}(p k, s)=$ hash $\left(m^{\prime}\right)$ succeeds, the process performs an array lookup: it looks for an index $u$ in $[1, q S]$ such that $m[u]$ is defined and $m^{\prime}=m[u]$. If such an $u$ is found, that is, $m^{\prime}$ has already been received by the signing oracle, we simply end the oracle. Otherwise, we execute the event forge and implicitly end the oracle. Arrays and array lookups are crucial in this calculus, and will help to model many properties which were hard to capture.

Finally, we add a hash oracle, which is similar to the signing oracle $O S$ but returns the hash of the message instead of its signature:

$$
\text { foreach } i H \leq q H \text { do } O H[i H](x[i H]: \text { bitstring }):=\operatorname{return}(\operatorname{hash}(x[i H]))
$$

To lighten the notation, some array indexes can be omitted in the input we give to our prover. Precisely, when $x$ is defined under foreach $i_{1} \leq n_{1} \ldots$ foreach $i_{m} \leq$ $n_{m}, x$ is always an array with indexes $i_{1}, \ldots, i_{m}$, so we abbreviate all occurrences of $x\left[i_{1}, \ldots, i_{m}\right]$ by $x$. Here, all array indexes in $O S$ and $O H$ can then be omitted.

We can remark that the signature and test oracles only make sense after the generation oracle Ogen has been called, since they make use of the keys $p k$ and sk computed by $O g e n$. So we define $O S$ and $O T$ after $O g e n$ by a sequential composition. In contrast, $O S$ and $O T$ are simultaneously available, so we use a parallel composition $Q_{S} \mid Q_{T}$ where $Q_{S}$ and $Q_{T}$ are the processes (11) and (2) respectively. Similarly, $O H$ is composed in parallel with the rest of the process. So we obtain the following game which models the security of the FDH signature scheme in the random oracle model:

$$
\begin{gathered}
G_{0}=\text { foreach } i H \leq q H \text { do } O H(x: \text { bitstring }):=\operatorname{return}(\operatorname{hash}(x)) \\
\mid \text { Ogen }():=r \stackrel{R}{\leftarrow} \text { seed; } p k \leftarrow \operatorname{pkgen}(r) ; s k \leftarrow \operatorname{skgen}(r) ; \operatorname{return}(p k) ; \\
(\text { foreach } i S \leq q S \text { do } O S(m: \text { bitstring }):=\operatorname{return}(\operatorname{invf}(s k, \operatorname{hash}(m))) \\
\mid O T\left(m^{\prime}: \text { bitstring, } s: D\right):=\operatorname{if} \mathrm{f}(p k, s)=\operatorname{hash}\left(m^{\prime}\right) \text { then } \\
\text { find } u \leq q S \text { suchthat }\left(\operatorname{defined}(m[u]) \wedge m^{\prime}=m[u]\right) \text { then end } \\
\text { else event forge) }
\end{gathered}
$$

Our calculus obviously also has a construct for calling oracles. However, we do not need it explicitly in this paper, because oracles are called by the adversary, not by processes we write ourselves.

As detailed in [13]14], we require some well-formedness invariants to guarantee that several definitions of the same oracle cannot be simultaneously available, that bit-strings are of their expected type, and that arrays are used properly (that each cell of an array is assigned at most once during execution, and that variables are accessed only after being initialized). The formal semantics of the calculus can be found in $[13$.

\subsection{Observational Equivalence}

We denote by $\operatorname{Pr}[Q \rightsquigarrow a]$ the probability that the answer of $Q$ to the oracle call Ostart() is a, where Ostart is an oracle called to start the experiment. We 
denote by $\operatorname{Pr}[Q \rightsquigarrow \mathcal{E}]$ the probability that the process $Q$ executes exactly the sequence of events $\mathcal{E}$, in the order of $\mathcal{E}$, when oracle Ostart() is called.

In the next definition, we use a context $C$ to represent an algorithm that tries to distinguish $Q$ from $Q^{\prime}$. A context $C$ is put around a process $Q$ by $C[Q]$. This construct means that $Q$ is put in parallel with some other process $Q^{\prime}$ contained in $C$, possibly hiding some oracles defined in $Q$, so that, when considering $C^{\prime}[C[Q]]$, $C^{\prime}$ cannot call these oracles. This will be detailed in the following of this section.

Definition 1 (Observational equivalence). Let $Q$ and $Q^{\prime}$ be two processes that satisfy the well-formedness invariants.

$A$ context $C$ is said to be acceptable for $Q$ if and only if $C$ does not contain events, $C$ and $Q$ have no common variables, and $C[Q]$ satisfies the wellformedness invariants.

We say that $Q$ and $Q^{\prime}$ are observationally equivalent up to probability $p$, written $Q \approx_{p} Q^{\prime}$, when for all $t$, for all contexts $C$ acceptable for $Q$ and $Q^{\prime}$ that run in time at most $t$, for all bit-strings a, $\left|\operatorname{Pr}[C[Q] \rightsquigarrow a]-\operatorname{Pr}\left[C\left[Q^{\prime}\right] \rightsquigarrow a\right]\right| \leq p(t)$ and $\sum_{\mathcal{E}}\left|\operatorname{Pr}[C[Q] \rightsquigarrow \mathcal{E}]-\operatorname{Pr}\left[C\left[Q^{\prime}\right] \rightsquigarrow \mathcal{E}\right]\right| \leq p(t)$.

This definition formalizes that the probability that an algorithm $C$ running in time $t$ distinguishes the games $Q$ and $Q^{\prime}$ is at most $p(t)$. The context $C$ is not allowed to access directly the variables of $Q$ (using find). We say that a context $C$ runs in time $t$, when for all processes $Q$, the time spent in $C$ in any trace of $C[Q]$ is at most $t$, ignoring the time spent in $Q$. (The runtime of a context is bounded. Indeed, we bound the length of messages in calls or returns to oracle $O$ by a value maxlen $\left(O, \arg _{i}\right)$ or maxlen $\left(O, \operatorname{res}_{i}\right)$. Longer messages are truncated. The length of random numbers created by $C$ is bounded; the number of instructions executed by $C$ is bounded; and the time of a function evaluation is bounded by a function of the length of its arguments.)

Definition 2. We say that $Q$ executes event $e$ with probability at most $p$ if and only if for all $t$, for all contexts $C$ acceptable for $Q$ that run in time $t$, $\sum_{\mathcal{E}, e \in \mathcal{E}} \operatorname{Pr}[C[Q] \rightsquigarrow \mathcal{E}] \leq p(t)$.

The above definitions allow us to perform proofs using sequences of indistinguishable games. The following lemma is straightforward:

Lemma 1. 1. $\approx_{p}$ is reflexive and symmetric.

2. If $Q \approx_{p} Q^{\prime}$ and $Q^{\prime} \approx_{p^{\prime}} Q^{\prime \prime}$, then $Q \approx_{p+p^{\prime}} Q^{\prime \prime}$.

3. If $Q$ executes event $e$ with probability at most $p$ and $Q \approx_{p^{\prime}} Q^{\prime}$, then $Q^{\prime}$ executes event $e$ with probability at most $p+p^{\prime}$.

4. If $Q \approx_{p} Q^{\prime}$ and $C$ is a context acceptable for $Q$ and $Q^{\prime}$ that runs in time $t_{C}$, then $C[Q] \approx_{p^{\prime}} C\left[Q^{\prime}\right]$ where $p^{\prime}(t)=p\left(t+t_{C}\right)$.

5. If $Q$ executes event $e$ with probability at most $p$ and $C$ is a context acceptable for $Q$ that runs in time $t_{C}$, then $C[Q]$ executes event e with probability at most $p^{\prime}$ where $p^{\prime}(t)=p\left(t+t_{C}\right)$.

Properties 2 and 3 are key to computing probabilities coming from a sequence of games. Indeed, our prover will start from a game $G_{0}$ corresponding to the 
initial attack, and build a sequence of observationally equivalent games $G_{0} \approx_{p_{1}}$ $G_{1} \approx_{p_{2}} \ldots \approx_{p_{m}} G_{m}$. By Property 2, we conclude that $G_{0} \approx_{p_{1}+\ldots+p_{m}} G_{m}$. By Property 3, we can bound the probability that $G_{0}$ executes an event from the probability that $G_{m}$ executes this event.

The elementary transformations used to build each game from the previous one can in particular come from an algorithmic assumption on a cryptographic primitive. This assumption needs to be specified as an observational equivalence $L \approx_{p} R$. To use it to transform a game $G$, the prover finds a context $C$ such that $G \approx_{0} C[L]$ by purely syntactic transformations, and builds a game $G^{\prime}$ such that $G^{\prime} \approx_{0} C[R]$ by purely syntactic transformations. $C$ is the simulator usually defined for reductions. By Property 4 , we have $C[L] \approx_{p^{\prime}} C[R]$, so $G \approx_{p^{\prime}} G^{\prime}$. The context $C$ typically hides the oracles of $L$ and $R$ so that they are visible from $C$ but not from the adversary $C^{\prime}$ against $G \approx_{p^{\prime}} G^{\prime}$. The context $C^{\prime}[C[]]$ then defines the adversary against the algorithmic assumption $L \approx_{p} R$.

If the security assumptions are initially not in the form of an equivalence $L \approx_{p} R$, one needs to manually prove such an equivalence that formalizes the desired security assumption. The design of such equivalences can be delicate, but this is a one-time effort: the same equivalence can be reused for proofs that rely on the same assumption. For instance, we give below such an equivalence for one-wayness, and use it not only for the proof of the FDH signature scheme, but also for proofs of encryption schemes as mentioned in Section 4.2. Similarly, the definition of security of a signature (UF-CMA) says that some event is executed with negligible probability. When we want to prove the security of a protocol using a signature scheme, we use a manual proof of an equivalence that corresponds to that definition, done once for UF-CMA in the long version of this paper [15].

The prover automatically establishes certain equivalences $G_{0} \approx_{p} G_{m}$ as mentioned above. However, the user can give only the left-hand side of the equivalence $G_{0}$; the right-hand side $G_{m}$ is obtained by the prover. As a consequence, the prover is in general not appropriate for proving automatically properties $L \approx_{p} R$ in which $L$ and $R$ are both given a priori: the right-hand side found by the prover is unlikely to correspond exactly to the desired right-hand side. On the other hand, the prover can check security properties on the right-hand side $G_{m}$ it finds, for example that the event forge cannot be executed by $G_{m}$. Using $G_{0} \approx_{p} G_{m}$, it concludes that $G_{0}$ executes forge with probability at most $p$.

\section{Characterization of One-Wayness and Unforgeability}

In this section, we introduce the assumption (one-wayness) and the security notion (unforgeability) to achieve.

\subsection{Trapdoor One-Way Permutations}

Most cryptographic protocols rely on the existence of trapdoor one-way permutations. They are families of permutations, which are easy to compute, but hard to invert, unless one has a trapdoor. 
The Computational Model. A family of permutations $\mathcal{P}$ onto a set $D$ is defined by the three following algorithms:

- The key generation algorithm kgen (which can be split in two sub-algorithms pkgen and skgen). On input a seed $r$, the algorithm kgen produces a pair $(p k, s k)$ of matching public and secret keys. The public key $p k$ specifies the actual permutation $f_{p k}$ onto the domain $D$.

- The evaluation algorithm $\mathrm{f}$. Given a public key $p k$ and a value $x \in D$, it outputs $y=f_{p k}(x)$.

- The inversion algorithm invf. Given an element $y$, and the trapdoor sk, invf outputs the unique pre-image $x$ of $y$ with respect to $f_{p k}$.

The above properties simply require the algorithms to be efficient. The "onewayness" property is more intricate, since it claims the "non-existence" of some efficient algorithm: one wants that the success probability of any adversary $\mathcal{A}$ within a reasonable time is small, where this success is commonly defined by

$$
\operatorname{Succ}_{\mathcal{P}}^{\text {ow }}(\mathcal{A})=\operatorname{Pr}\left[\begin{array}{l}
r \stackrel{R}{\leftarrow} \text { seed },(p k, s k) \leftarrow \operatorname{kgen}(r), x \stackrel{R}{\leftarrow} D, y \leftarrow \mathrm{f}(p k, x), \\
x^{\prime} \leftarrow \mathcal{A}(p k, y): x=x^{\prime}
\end{array}\right] .
$$

Eventually, we denote by $\operatorname{Succ}_{\mathcal{P}}^{\text {ow }}(t)$ the maximal success probability an adversary can get within time $t$.

Syntactic Rules. Let seed be a large, fixed-length type, pkey, skey, and $D$ the types of public keys, secret keys, and the domain of the permutations respectively. A family of trapdoor one-way permutations can then be defined as a set of four function symbols: skgen : seed $\rightarrow$ skey generates secret keys; pkgen : seed $\rightarrow$ pkey generates public keys; $\mathrm{f}:$ pkey $\times D \rightarrow D$ and invf : skey $\times D \rightarrow D$, such that, for each $p k, x \mapsto \mathrm{f}(p k, x)$ is a permutation of $D$, whose inverse permutation is $x \mapsto \operatorname{invf}(s k, x)$ when $p k=\operatorname{pkgen}(r)$ and $s k=\operatorname{skgen}(r)$.

The one-wayness property can be formalized in our calculus by requiring that $L R$ executes event invert with probability at $\operatorname{most} \operatorname{Succ}_{\mathcal{P}}^{\text {ow }}(t)$ in the presence of a context that runs in time $t$, where

$$
\begin{aligned}
L R= & \operatorname{Ogen}():=r_{0} \stackrel{R}{\leftarrow} \text { seed } ; x_{0} \stackrel{R}{\leftarrow} D ; \operatorname{return}\left(\operatorname{pkgen}\left(r_{0}\right), \mathrm{f}\left(\operatorname{pkgen}\left(r_{0}\right), x_{0}\right)\right) ; \\
& \operatorname{Oeq}\left(x^{\prime}: D\right):=\text { if } x^{\prime}=x_{0} \text { then event invert }
\end{aligned}
$$

Indeed, the event invert is executed when the adversary, given the public key $\operatorname{pkgen}\left(r_{0}\right)$ and the image of some $x_{0}$ by $\mathrm{f}$, manages to find $x_{0}$ (without having the trapdoor).

In order to use the one-wayness property in proofs of protocols, our prover needs a more general formulation of one-wayness, using "observationally equivalent" processes. We thus define two processes which are actually equivalent unless $L R$ executes event invert. We prove in the long version of this paper [15] the equivalence of Figure 1 where $p^{\text {ow }}(t)=n_{\mathrm{k}} \times n_{\mathrm{f}} \times \operatorname{Succ}_{\mathcal{P}}^{\text {ow }}\left(t+\left(n_{\mathrm{k}} n_{\mathrm{f}}-1\right) t_{\mathrm{f}}+\left(n_{\mathrm{k}}-\right.\right.$ 1) $\left.t_{\text {pkgen }}\right), t_{\mathrm{f}}$ is the time of one evaluation of $\mathrm{f}$, and $t_{\text {pkgen }}$ is the time of one evaluation of pkgen. In this equivalence, the function symbols pkgen' $:$ seed $\rightarrow$ pkey and 


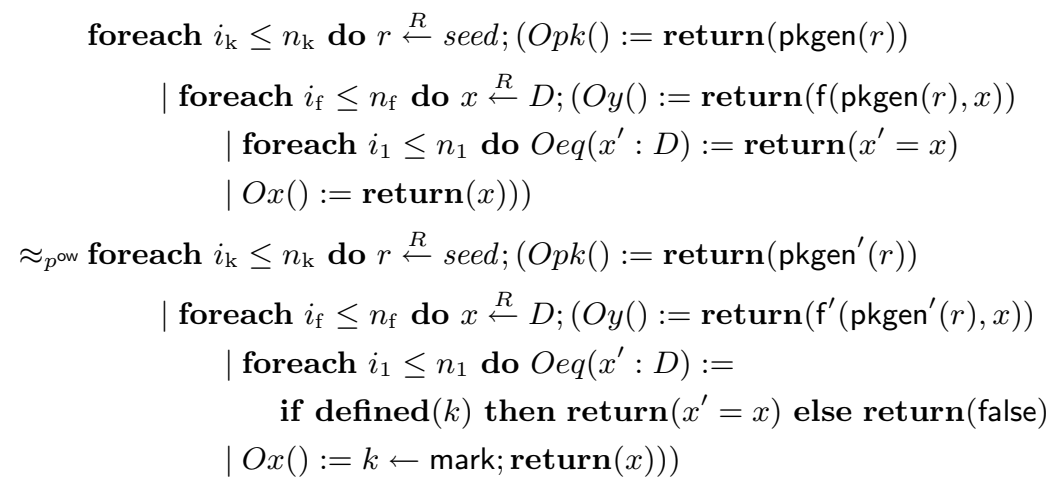

Fig. 1. Definition of one-wayness

$\mathrm{f}^{\prime}:$ pkey $\times D \rightarrow D$ are such that the functions associated to the primed symbols pkgen $^{\prime}, f^{\prime}$ are equal to the functions associated to their corresponding unprimed symbol pkgen, $f$, respectively. We replace pkgen and $f$ with pkgen ${ }^{\prime}$ and $f^{\prime}$ in the right-hand side just to prevent repeated applications of the transformation with the same keys, which would lead to an infinite loop.

In this equivalence, we consider $n_{\mathrm{k}}$ keys $\operatorname{pkgen}\left(r\left[i_{\mathrm{k}}\right]\right)$ instead of a single one, and $n_{\mathrm{f}}$ antecedents of $\mathrm{f}$ for each key, $x\left[i_{\mathrm{k}}, i_{\mathrm{f}}\right]$. The first oracle $O p k\left[i_{\mathrm{k}}\right]$ publishes the public key pkgen $\left(r\left[i_{\mathrm{k}}\right]\right)$. The second group of oracles first picks a new $x\left[i_{\mathrm{k}}, i_{\mathrm{f}}\right]$, and then makes available three oracles: $O y\left[i_{\mathrm{k}}, i_{\mathrm{f}}\right]$ returns the image of $x\left[i_{\mathrm{k}}, i_{\mathrm{f}}\right]$ by $\mathrm{f}, \operatorname{Oeq}\left[i_{\mathrm{k}}, i_{\mathrm{f}}, i_{1}\right]$ returns true when it receives $x\left[i_{\mathrm{k}}, i_{\mathrm{f}}\right]$ as argument, and $O x\left[i_{\mathrm{k}}, i_{\mathrm{f}}\right]$ returns $x\left[i_{\mathrm{k}}, i_{\mathrm{f}}\right]$ itself. The one-wayness property guarantees that when $O x\left[i_{\mathrm{k}}, i_{\mathrm{f}}\right]$ has not been called, the adversary has little chance of finding $x\left[i_{\mathrm{k}}, i_{\mathrm{f}}\right]$, so $O e q\left[i_{\mathrm{k}}, i_{\mathrm{f}}, i_{1}\right]$ returns false. Therefore, we can replace the left-hand side of the equivalence with its right-hand side, in which $O x\left[i_{\mathrm{k}}, i_{\mathrm{f}}\right]$ records that it has been called by defining $k\left[i_{\mathrm{k}}, i_{\mathrm{f}}\right]$, and $O e q\left[i_{\mathrm{k}}, i_{\mathrm{f}}, i_{1}\right]$ always returns false when $k\left[i_{\mathrm{k}}, i_{\mathrm{f}}\right]$ is not defined, that is, when $O x\left[i_{\mathrm{k}}, i_{\mathrm{f}}\right]$ has not been called.

In the left-hand side of the equivalences used to specify primitives, the oracles must consist of a single return instruction. This restriction allows us to model many equivalences that define cryptographic primitives, and it simplifies considerably the transformation of processes compared to using the general syntax of processes. (In order to use an equivalence $L \approx_{p} R$, we need to recognize processes that can easily be transformed into $C[L]$ for some context $C$, to transform them into $C[R]$. This is rather easy to do with such oracles: we just need to recognize terms that occur as a result of these oracles. That would be much more difficult with general processes.)

Since $x \mapsto \mathrm{f}(\operatorname{pkgen}(r), x)$ and $x \mapsto \operatorname{invf}(\operatorname{skgen}(r), x)$ are inverse permutations, we have:

$$
\forall r: \text { seed, } \forall x: D, \operatorname{invf}(\operatorname{skgen}(r), \mathrm{f}(\operatorname{pkgen}(r), x))=x
$$

Since $x \mapsto \mathrm{f}(p k, x)$ is injective, $\mathrm{f}(p k, x)=\mathrm{f}\left(p k, x^{\prime}\right)$ if and only if $x=x^{\prime}$ :

$$
\forall p k: p k e y, \forall x: D, \forall x^{\prime}: D,\left(\mathrm{f}(p k, x)=\mathrm{f}\left(p k, x^{\prime}\right)\right)=\left(x=x^{\prime}\right)
$$


Since $x \mapsto \mathrm{f}(p k, x)$ is a permutation, when $x$ is a uniformly distributed random number, we can replace $x$ with $\mathrm{f}(p k, x)$ everywhere, without changing the probability distribution. In order to enable automatic proof, we give a more restricted formulation of this result:

$$
\text { foreach } i_{\mathrm{k}} \leq n_{\mathrm{k}} \text { do } r \stackrel{R}{\leftarrow} \operatorname{seed} ;(\operatorname{Opk}():=\operatorname{return}(\operatorname{pkgen}(r))
$$

| foreach $i_{\mathrm{f}} \leq n_{\mathrm{f}}$ do $x \stackrel{R}{\leftarrow} D ;(\operatorname{Oant}():=\operatorname{return}(\operatorname{invf}(\operatorname{skgen}(r), x))$

$$
\mid \operatorname{Oim}():=\operatorname{return}(x)))
$$

$\approx_{0}$ foreach $i_{\mathrm{k}} \leq n_{\mathrm{k}}$ do $r \stackrel{R}{\leftarrow} \operatorname{seed} ;(\operatorname{Opk}():=\operatorname{return}(\operatorname{pkgen}(r))$

$$
\begin{aligned}
\mid \text { foreach } i_{\mathrm{f}} \leq n_{\mathrm{f}} \text { do } x \stackrel{R}{\leftarrow} D ;(\operatorname{Oant}(): & :=\operatorname{return}(x) \\
\mid \operatorname{Oim}(): & =\operatorname{return}(\mathrm{f}(\operatorname{pkgen}(r), x))))
\end{aligned}
$$

which allows to perform the previous replacement only when $x$ is used in calls to invf(skgen $(r), x)$, where $r$ is a random number such that $r$ occurs only in pkgen $(r)$ and invf(skgen $(r), x)$ for some random numbers $x$.

\subsection{Signatures}

The Computational Model. A signature scheme $\mathrm{S}=$ (kgen, sign, verify) is defined by:

- The key generation algorithm kgen (which can be split in two sub-algorithms pkgen and skgen). On input a random seed $r$, the algorithm kgen produces a pair $(p k, s k)$ of matching keys.

- The signing algorithm sign. Given a message $m$ and a secret key sk, sign produces a signature $\sigma$. For sake of clarity, we restrict ourselves to the deterministic case.

- The verification algorithm verify. Given a signature $\sigma$, a message $m$, and a public key $p k$, verify tests whether $\sigma$ is a valid signature of $m$ with respect to $p k$.

We consider here (existential) unforgeability under adaptive chosen-message attack (UF-CMA) [23], that is, the attacker can ask the signer to sign any message of its choice, in an adaptive way, and has to provide a signature on a new message. In its answer, there is indeed the natural restriction that the returned message has not been asked to the signing oracle.

When one designs a signature scheme, one wants to computationally rule out existential forgeries under adaptive chosen-message attacks. More formally, one wants that the success probability of any adversary $\mathcal{A}$ with a reasonable time is small, where

$$
\operatorname{Succ}_{\mathrm{S}}^{\text {uf }-\mathrm{cma}}(\mathcal{A})=\operatorname{Pr}\left[\begin{array}{l}
r \stackrel{R}{\leftarrow} \operatorname{seed},(p k, s k) \leftarrow \operatorname{kgen}(r),(m, \sigma) \leftarrow \mathcal{A}^{\operatorname{sign}(\cdot, s k)}(p k): \\
\operatorname{verify}(m, p k, \sigma)=1
\end{array}\right] .
$$


As above, we denote by $\operatorname{Succ}_{\mathrm{S}}^{\mathrm{uf}-\mathrm{cma}}\left(n_{\mathrm{s}}, \ell, t\right)$ the maximal success probability an adversary can get within time $t$, after at most $n_{\mathrm{s}}$ queries to the signing oracle, where the maximum length of all messages in queries is $\ell$.

Syntactic Rules. Let seed be a large, fixed-length type. Let pkey, skey, and signature the types of public keys, secret keys, and signatures respectively. A signature scheme is defined as a set of four function symbols: skgen : seed $\rightarrow$ skey generates secret keys; pkgen : seed $\rightarrow$ pkey generates public keys; sign : bitstring $\times$ skey $\rightarrow$ signature generates signatures; and verify : bitstring $\times$ pkey $\times$ signature $\rightarrow$ bool verifies signatures.

The signature verification succeeds for signatures generated by sign, that is,

$$
\forall m \text { : bitstring, } \forall r: \text { seed, } \operatorname{verify}(m, \operatorname{pkgen}(r), \operatorname{sign}(m, \operatorname{skgen}(r)))=\text { true }
$$

According to the previous definition of UF-CMA, the following process $L R$ executes event forge with probability at most $\operatorname{Succ}_{\mathrm{S}}^{\mathrm{uf}-\mathrm{cma}}\left(n_{\mathrm{s}}, \ell, t\right)$ in the presence of a context that runs in time $t$, where

$$
\begin{gathered}
L R=O g e n():=r \stackrel{R}{\leftarrow} \text { seed } ; p k \leftarrow \operatorname{pkgen}(r) ; s k \leftarrow \operatorname{skgen}(r) ; \operatorname{return}(p k) ; \\
\left(\text { foreach } i_{\mathrm{s}} \leq n_{\mathrm{s}} \text { do } O S(m: \text { bitstring }):=\operatorname{return}(\operatorname{sign}(m, s k))\right. \\
\mid O T\left(m^{\prime}: \text { bitstring, } s: \text { signature }\right):=\text { if } \operatorname{verify}\left(m^{\prime}, p k, s\right) \text { then } \\
\quad \text { find } u_{\mathrm{s}} \leq n_{\mathrm{s}} \text { suchthat }\left(\operatorname{defined}\left(m\left[u_{\mathrm{s}}\right]\right) \wedge m^{\prime}=m\left[u_{\mathrm{s}}\right]\right) \\
\quad \text { then end else event forge })
\end{gathered}
$$

and $\ell$ is the maximum length of $m$ and $m^{\prime}$. This is indeed clear since event forge is raised if a signature is accepted (by the verification algorithm), while the signing algorithm has not been called on the signed message.

\section{Examples}

\subsection{FDH Signature}

The Full-Domain Hash (FDH) signature scheme 9] is defined as follows: Let pkgen, skgen, $f$, invf define a family of trapdoor one-way permutations. Let hash be a hash function, in the random oracle model. The FDH signature scheme uses the functions pkgen and skgen as key-generation functions, the signing $\operatorname{algorithm}$ is $\operatorname{sign}(m, s k)=\operatorname{invf}(s k$, hash $(m))$, and the verification algorithm is $\operatorname{verify}\left(m^{\prime}, p k, s\right)=\left(\mathrm{f}(p k, s)=\operatorname{hash}\left(m^{\prime}\right)\right)$. In this section, we explain how our automatic prover finds the well-known bound for $\mathrm{Succ}_{\mathrm{S}}^{\text {uf }- \text { cma }}$ for the FDH signature scheme.

The input given to the prover contains two parts. First, it contains the definition of security of primitives used to build the FDH scheme, that is, the definition of one-way trapdoor permutations (3), (4), (5), and (6) as detailed 
in Section 3.1 and the formalization of a hash function in the random oracle model:

foreach $i_{\mathrm{h}} \leq n_{\mathrm{h}}$ do $O H(x$ : bitstring $):=\operatorname{return}(\operatorname{hash}(x))[$ all $]$

$\approx_{0}$ foreach $i_{\mathrm{h}} \leq n_{\mathrm{h}}$ do $O H(x:$ bitstring $):=$

find $u \leq n_{\mathrm{h}}$ suchthat $(\operatorname{defined}(x[u], r[u]) \wedge x=x[u])$ then $\operatorname{return}(r[u])$

else $r \stackrel{R}{\leftarrow} D ; \operatorname{return}(r)$

This equivalence expresses that we can replace a call to a hash function with a random oracle, that is, an oracle that returns a fresh random number when it is called with a new argument, and the previously returned result when it is called with the same argument as in a previous call. Such a random oracle is implemented in our calculus by a lookup in the array $x$ of the arguments of hash. When a $u$ such that $x[u], r[u]$ are defined and $x=x[u]$ is found, hash has already been called with $x$, at call number $u$, so we return the result of that call, $r[u]$. Otherwise, we create a fresh random number $r$. (The indication $[a l l]$ on the first line of (8) instructs the prover to replace all occurrences of hash in the game.)

Second, the input file contains as initial game the process $G_{0}$ of Section 2.1. As detailed in Section 3.2, this game corresponds to the definition of security of the FDH signature scheme (7). An important remark is that we need to add to the standard definition of security of a signature scheme the hash oracle. This is necessary so that, after transformation of hash into a random oracle, the adversary can still call the hash oracle. (The adversary does not have access to the arrays that encode the values of the random oracle.) Our goal is to bound the probability $p(t)$ that event forge is executed in this game in the presence of a context that runs in time $t: p(t)=\operatorname{Succ}_{\mathrm{S}}^{\mathrm{uf}-\mathrm{cma}}\left(q S, \ell, t+t_{H}\right) \geq \operatorname{Succ}_{\mathrm{S}}^{\text {uf }-\mathrm{cma}}(q S, \ell, t)$ where $t_{H}$ is the total time spent in the hash oracle and $\ell$ is the maximum length of $m$ and $m^{\prime}$.

Given this input, our prover automatically produces a proof that this game executes event forge with probability $p(t) \leq(q H+q S+1) \operatorname{Succ}_{\mathcal{P}}^{\text {ow }}(t+(q H+$ $\left.q S) t_{\mathrm{f}}+\left(3 q S+2 q H+q S^{2}+2 q S q H+q H^{2}\right) t_{\mathrm{eq}}(\ell)\right)$ where $\ell$ is the maximum length of a bit-string in $m, m^{\prime}$, or $x$ and $t_{\mathrm{eq}}(\ell)$ is the time of a comparison between bit-strings of length at most $\ell$. (Evaluating a find implies evaluating the condition of the find for each value of the indexes, so here the lookup in an array of size $n$ of bit-strings of length $\ell$ is considered as taking time $n \times$ $t_{\text {eq }}(\ell)$, although there are in fact more efficient algorithms for this particular case of array lookup.) If we ignore the time of bit-string comparisons, we obtain the usual upper-bound [10] $(q H+q S+1) \operatorname{Succ}_{\mathcal{P}}^{\text {ow }}\left(t+(q H+q S) t_{\mathrm{f}}\right)$. The prover also outputs the sequence of games that leads to this proof, and a succinct explanation of the transformation performed between consecutive games of the sequence. The input and output of the prover, as well as the prover itself, are available at http://www.di.ens.fr/ planchet/cryptoc/FDH/; the runtime of the prover on this example is $14 \mathrm{~ms}$ on a Pentium M $1.8 \mathrm{GHz}$. The prover has been implemented in Ocaml and contains 14800 lines of code. 
We sketch here the main proof steps. Starting from the initial game $G_{0}$ given in Section 2.1. the prover tries to apply all observational equivalences it has as hypotheses, that is here, (3), (6), and (8). It succeeds applying the security of the hash function (8), so it transforms the game accordingly, by replacing the left-hand side with the right-hand side of the equivalence. Each call to hash is then replaced with a lookup in the arguments of all calls to hash. When the argument of hash is found in one of these arrays, the returned result is the same as the result previously returned by hash. Otherwise, we pick a fresh random number and return it.

The obtained game is then simplified. In particular, when the argument $m^{\prime}$ of $O T$ is found in the arguments $m$ of the call to hash in $O S$, the find in $O T$ always succeeds, so its else branch can be removed (that is, when $m^{\prime}$ has already been passed to the signature oracle, it is not a forgery).

Then, the prover tries to apply an observational equivalence. All transformations fail, but when applying (6), the game contains $\operatorname{invf}(s k, y)$ while (6) expects invf(skgen $(r), y)$, which suggests to remove assignments to variable $s k$ for it to succeed. So the prover performs this removal: it substitutes $\operatorname{skgen}(r)$ for $s k$ and removes the assignment $s k \leftarrow \operatorname{skgen}(r)$. The transformation (6) is then retried. It now succeeds, which leads to replacing $r_{j}$ with $\mathrm{f}\left(\operatorname{pkgen}(r), r_{j}\right)$ and $\operatorname{invf}\left(\operatorname{skgen}(r), r_{j}\right)$ with $r_{j}$, where $r_{j}$ represents the random numbers that are the result of the random oracle. (The term $\mathrm{f}\left(\operatorname{pkgen}(r), r_{j}\right)$ can then be computed by oracle $O y$ of (3) and $r_{j}$ can be computed by $O x$.) More generally, in our prover, when a transformation $\mathcal{T}$ fails, it may return transformations $\mathcal{T}^{\prime}$ to apply in order to enable $\mathcal{T}$ [14, Section 5]. In this case, the prover applies the suggested transformations $\mathcal{T}^{\prime}$ and retries the transformation $\mathcal{T}$.

The obtained game is then simplified. In particular, by injectivity of $f(5)$, the prover replaces terms of the form $\mathrm{f}(p k, s)=\mathrm{f}\left(\operatorname{pkgen}(r), r_{j}\right)$ with $s=r_{j}$, knowing $p k=\operatorname{pkgen}(r)$. (The test $s=r_{j}$ can then be computed by oracle Oeq of (3) $)$.)

The prover then tries to apply an observational equivalence. It succeeds using the definition of one-wayness (3). This transformation leads to replacing $\mathrm{f}\left(\operatorname{pkgen}(r), r_{j}\right)$ with $\mathrm{f}^{\prime}\left(\operatorname{pkgen}^{\prime}(r), r_{j}\right), r_{j}$ with $k_{j} \leftarrow$ mark; $r_{j}$, and $s=r_{j}$ with find $u_{j} \leq N$ suchthat (defined $\left(k_{j}\left[u_{j}\right]\right) \wedge$ true) then $s=r_{j}$ else false. The difference of probability is $p^{\text {ow }}\left(t+t^{\prime}\right)=n_{\mathrm{k}} \times n_{\mathrm{f}} \times \operatorname{Succ}_{\mathcal{P}}^{\text {ow }}\left(t+t^{\prime}+\left(n_{\mathrm{k}} n_{\mathrm{f}}-1\right) t_{\mathrm{f}}+\right.$ $\left.\left(n_{\mathrm{k}}-1\right) t_{\mathrm{pkgen}}\right)=(q H+q S+1) \operatorname{Succ}_{\mathcal{P}}^{\text {ow }}\left(t+t^{\prime}+(q H+q S) t_{\mathrm{f}}\right)$ where $n_{\mathrm{k}}=1$ is the number of key pairs considered, $n_{\mathrm{f}}=q H+q S+1$ is the number of antecedents of $\mathrm{f}$, and $t^{\prime}=\left(3 q S+2 q H+q S^{2}+2 q S q H+q H^{2}\right) t_{\text {eq }}(\ell)$ is the runtime of the context put around the equivalence (3).

Finally, the obtained game is simplified again. Thanks to some equational reasoning, the prover manages to show that the find in $O T$ always succeeds, so its else branch can be removed. The prover then detects that the forge event cannot be executed in the resulting game, so the desired property is proved, and the probability that forge is executed in the initial game is the sum of the differences of probability between games of the sequence, which here comes only from the application of one-wayness (3). 


\subsection{Encryption Schemes}

Besides proving the security of many protocols [14, we have also used our prover for proving other cryptographic schemes. For example, our prover can show that the basic Bellare-Rogaway construction [9] without redundancy (i.e. $\mathcal{E}(m, r)=$ $\mathrm{f}(r) \|$ hash $(r)$ xor $m)$ is IND-CPA, with the following manual proof:

$\begin{array}{ll}\text { crypto hash } & \text { apply the security of hash (8) } \\ \text { remove_assign binder pk remove assignments to } p k \\ \text { crypto } \mathrm{f} r & \text { apply the security of } \mathrm{f}(\mathrm{3}) \text { with random seed } r \\ \text { crypto xor } * & \text { apply the security of xor as many times as possible } \\ \text { success } & \text { check that the desired property is proved }\end{array}$

These manual indications are necessary because (3) can also be applied without removing the assignments to $p k$, but with different results: $\mathrm{f}(p k, x)$ is computed by applying $f$ to the results of oracles $O p k$ and $O x$ if assignments to $p k$ are not removed, and by oracle $O y$ if assignments to $p k$ are removed.

With similar manual indications, it can show that the enhanced variant with redundancy $\mathcal{E}(m, r)=\mathrm{f}(r) \|$ hash $(r)$ xor $m \|$ hash' $^{\prime}$ hash $(r)$ xor $\left.m, r\right)$ is IND-CCA2. With an improved treatment of the equational theory of xor, we believe that it could also show that $\mathcal{E}(m, r)=\mathrm{f}(r) \|$ hash $(r)$ xor $m \|$ hash $^{\prime}(m, r)$ is IND-CCA2.

\section{Conclusion}

We have presented a new tool to automatically prove the security of both cryptographic primitives and cryptographic protocols. As usual, assumptions and expected security notions have to be stated. For the latter, specifications are quite similar to the usual definitions, where a "bad" event has to be shown to be unlikely. However, the former may seem more intricate, since it has to be specified as an observational equivalence. Anyway, this has to be done only once for all proofs, and several specifications have already been given in [131415]: one-wayness, UF-CMA signatures, UF-CMA message authentication codes, INDCPA symmetric stream ciphers, IND-CPA and IND-CCA2 public-key encryption, hash functions in the random oracle model, xor, with detailed proofs for the first three. Thereafter, the protocol/scheme itself has to be specified, but the syntax is quite close to the notations classically used in cryptography. Eventually, the prover provides the sequence of transformations, and thus of games, which lead to a final experiment (indistinguishable from the initial one) in which the "bad" event never appears. Since several paths may be used for such a sequence, the user is allowed (but does not have) to interact with the prover, in order to make it follow a specific sequence. Of course, the prover will accept only if the sequence is valid. Contrary to most of the formal proof techniques, the failure of the prover does not lead to an attack. It just means that the prover did not find an appropriate sequence of games.

Acknowledgments. We thank Jacques Stern for initiating our collaboration on this topic and the anonymous reviewers for their helpful comments. This work was partly supported by ARA SSIA Formacrypt. 


\section{References}

1. M. Abadi and P. Rogaway. Reconciling two views of cryptography (the computational soundness of formal encryption). Journal of Cryptology, 15(2):103-127, 2002 .

2. M. Backes and P. Laud. A mechanized, cryptographically sound type inference checker. In Workshop on Formal and Computational Cryptography (FCC'06), July 2006. To appear.

3. M. Backes and B. Pfitzmann. Symmetric encryption in a simulatable Dolev-Yao style cryptographic library. In CSF W'04. IEEE, June 2004.

4. M. Backes and B. Pfitzmann. Relating symbolic and cryptographic secrecy. In 26th IEEE Symposium on Security and Privacy, pages 171-182. IEEE, May 2005.

5. M. Backes, B. Pfitzmann, and M. Waidner. A composable cryptographic library with nested operations. In $C C S^{\prime} 03$, pages 220-230. ACM, Oct. 2003.

6. M. Backes, B. Pfitzmann, and M. Waidner. Symmetric authentication within a simulatable cryptographic library. In ESORICS'03, LNCS 2808, pages 271-290. Springer, Oct. 2003.

7. G. Barthe, J. Cederquist, and S. Tarento. A machine-checked formalization of the generic model and the random oracle model. In IJCAR'04, LNCS 3097, pages 385-399. Springer, July 2004.

8. M. Bellare. Practice-Oriented Provable Security. In ISW '97, LNCS 1396. Springer, 1997.

9. M. Bellare and P. Rogaway. Random Oracles Are Practical: a Paradigm for Designing Efficient Protocols. In CCS'93, pages 62-73. ACM Press, 1993.

10. M. Bellare and P. Rogaway. The Exact Security of Digital Signatures - How to Sign with RSA and Rabin. In Eurocrypt '96, LNCS 1070, pages 399-416. Springer, 1996.

11. M. Bellare and P. Rogaway. The Game-Playing Technique and its Application to Triple Encryption, 2004. Cryptology ePrint Archive 2004/331.

12. B. Blanchet. Automatic proof of strong secrecy for security protocols. In IEEE Symposium on Security and Privacy, pages 86-100, May 2004.

13. B. Blanchet. A computationally sound mechanized prover for security protocols. Cryptology ePrint Archive, Report 2005/401, Nov. 2005. Available at http://eprint.iacr.org/2005/401

14. B. Blanchet. A computationally sound mechanized prover for security protocols. In IEEE Symposium on Security and Privacy, pages 140-154, May 2006.

15. B. Blanchet and D. Pointcheval. Automated security proofs with sequences of games. Cryptology ePrint Archive, Report 2006/069, Feb. 2006. Available at http://eprint.iacr.org/2006/069

16. R. Canetti. Universally composable security: A new paradigm for cryptographic protocols. In FOCS'01, pages 136-145. IEEE, Oct. 2001. An updated version is available at Cryptology ePrint Archive, http://eprint.iacr.org/2000/067.

17. R. Canetti and J. Herzog. Universally composable symbolic analysis of cryptographic protocols (the case of encryption-based mutual authentication and key exchange). Cryptology ePrint Archive, Report 2004/334, 2004. Available at http://eprint.iacr.org/2004/334

18. V. Cortier and B. Warinschi. Computationally sound, automated proofs for security protocols. In ESOP'05, LNCS 3444, pages 157-171. Springer, Apr. 2005.

19. A. Datta, A. Derek, J. C. Mitchell, V. Shmatikov, and M. Turuani. Probabilistic polynomial-time semantics for a protocol security logic. In ICALP'05, LNCS 3580, pages 16-29. Springer, July 2005. 
20. W. Diffie and M. E. Hellman. New Directions in Cryptography. IEEE Transactions on Information Theory, IT-22(6):644-654, November 1976.

21. D. Dolev and A. C. Yao. On the Security of Public-Key Protocols. IEEE Transactions on Information Theory, 29(2):198-208, 1983.

22. S. Goldwasser and S. Micali. Probabilistic Encryption. Journal of Computer and System Sciences, 28:270-299, 1984.

23. S. Goldwasser, S. Micali, and R. Rivest. A Digital Signature Scheme Secure Against Adaptative Chosen-Message Attacks. SIAM Journal of Computing, 17(2):281-308, April 1988.

24. S. Halevi. A plausible approach to computer-aided cryptographic proofs. Cryptology ePrint Archive, Report 2005/181, June 2005. Available at http://eprint. iacr.org/2005/181

25. R. Janvier, Y. Lakhnech, and L. Mazaré. Completing the picture: Soundness of formal encryption in the presence of active adversaries. In ESOP'05, LNCS 3444, pages 172-185. Springer, Apr. 2005.

26. P. Laud. Handling encryption in an analysis for secure information flow. In ESOP'03, LNCS 2618, pages 159-173. Springer, Apr. 2003.

27. P. Laud. Symmetric encryption in automatic analyses for confidentiality against active adversaries. In IEEE Symposium on Security and Privacy, pages 71-85, May 2004.

28. P. Laud. Secrecy types for a simulatable cryptographic library. In $C C S^{\prime} 05$, pages 26-35. ACM, Nov. 2005.

29. P. D. Lincoln, J. C. Mitchell, M. Mitchell, and A. Scedrov. A probabilistic polytime framework for protocol analysis. In CCS'98, pages 112-121, Nov. 1998.

30. P. D. Lincoln, J. C. Mitchell, M. Mitchell, and A. Scedrov. Probabilistic polynomial-time equivalence and security protocols. In FM'99, LNCS 1708, pages 776-793. Springer, Sept. 1999.

31. P. Mateus, J. Mitchell, and A. Scedrov. Composition of cryptographic protocols in a probabilistic polynomial-time process calculus. In CONCUR 2003, LNCS 2761, pages 327-349. Springer, Sept. 2003.

32. D. Micciancio and B. Warinschi. Soundness of formal encryption in the presence of active adversaries. In TCC'04, LNCS 2951, pages 133-151. Springer, Feb. 2004.

33. J. C. Mitchell, A. Ramanathan, A. Scedrov, and V. Teague. A probabilistic polynomial-time calculus for the analysis of cryptographic protocols. Theoretical Computer Science, 353(1-3):118-164, Mar. 2006.

34. M. Naor and M. Yung. Universal One-Way Hash Functions and Their Cryptographic Applications. In STOC'89, pages 33-43. ACM Press, 1989.

35. C. Rackoff and D. R. Simon. Non-Interactive Zero-Knowledge Proof of Knowledge and Chosen Ciphertext Attack. In Crypto '91, LNCS 576, pages 433-444. Springer, 1992.

36. A. Ramanathan, J. Mitchell, A. Scedrov, and V. Teague. Probabilistic bisimulation and equivalence for security analysis of network protocols. In FOSSACS'04, LNCS 2987, pages 468-483. Springer, Mar. 2004.

37. V. Shoup. Sequences of games: a tool for taming complexity in security proofs, 2004. Cryptology ePrint Archive 2004/332.

38. C. Sprenger, M. Backes, D. Basin, B. Pfitzmann, and M. Waidner. Cryptographically sound theorem proving. In $C S F W^{\prime} 06$. IEEE, July 2006. To appear.

39. S. Tarento. Machine-checked security proofs of cryptographic signature schemes. In ESORICS'05, LNCS 3679, pages 140-158. Springer, Sept. 2005. 